\title{
The Environments of Cool Stars
}

\author{
Robert E. Stencel \\ Center for Astrophysics and Space Astronomy \\ University of Colorado \\ Boulder, CO 80309-0391 USA
}

\begin{abstract}
This review describes recent conclusions about the physical environment of red giant and supergiant stars. This includes coronae, chromospheres, dust formation and stellar winds. This knowledge can provide the boundary conditions for considering what role such objects play as members of binary star systems, where tidal forces and companion behavior alter observed characteristics.
\end{abstract}

\section{Observed Properties of Single Stars}

The study of binary stars can help us to understand the behavior of single stars by allowing for determination of stellar masses, internal structure (apsidal motion) and atmospheric properties (eclipses). In this review, I want to outline present knowledge concerning single post-main sequence late-type stars, so that we might better understand how the natural environment of cool stars can affect the interaction in binary stars. New multi-spectral observations and theoretical work is yielding more precise information about the boundary conditions involved with mass transfer and accretion.

Observationally, there are numerous aspects to the environments of cool stars. Ultimately, much of the phenomena is driven by interior conditions. S-type symbiotic systems often contain early $M$ giants which probably evolved vertically upward in the Hertzprung Russell Diagram (HRD), from solar-like main sequence stars. We have gained some insight into the atmospheric transformations associated with this evolution by presenting the statistical results of multi-spectral observations of cool stars on the HRD, and thereby illustrating several "dividing lines". These are indicative of atmospheric transitions between groups of stars which differ in their atmospheric diagnostics (Figure 1). Although represented in the HRD with narrow lines, the transitions are gradual and some admixture near the locus is found. More important than whether these lines are sharp or diffuse, is the significance of what they imply. A strong case can be made that we are observing the atmospheric responses to interior changes.

Access to the soft $x$-ray and ultraviolet regions of spectra has made possible the identification of the key dividing lines. Ayres et al. (1981), Haisch and Simon (1982) 


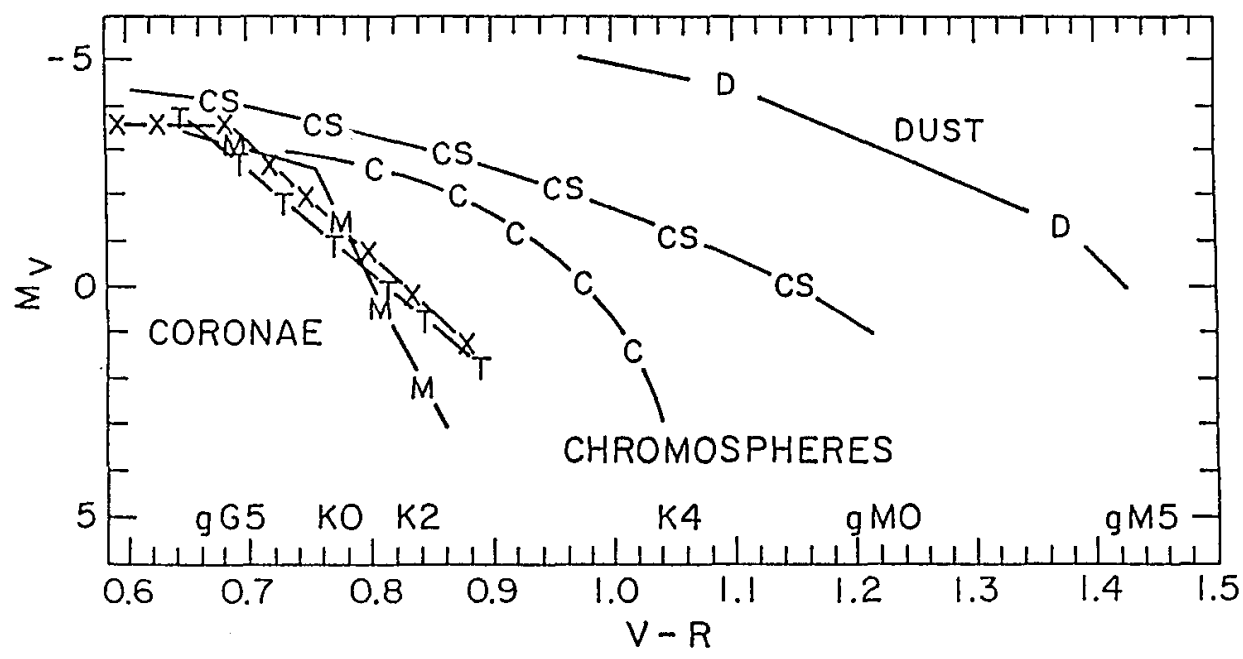

Figure 1: Divisions between major atmospheric conditions in the H-R Diagram. See text for identification of symbols.

and Gondoin et al. (1987) pointed out the rapid decrease in the soft x-ray flux with increasing $\mathrm{B}-\mathrm{V}$ color along the giant sequence (transition at $\mathrm{K} 1$ III, labelled $\mathrm{X}$ in Fig. 1). These authors argue that the soft $\mathrm{x}$-ray emission is a good diagnostic of solar-like coronal output (at temperatures of $10^{6-7} \mathrm{~K}$ ), and, therefore the lack of soft X-rays implies the lack of such coronal material among cooler giants. The coronal volume may be replaced by an "extended chromosphere" (Carpenter et al. 1985) dominated by fluorescent radiative processes (Judge 1986) among the cooler giants. O'Brien and Lambert (1986) have argued that this is indeed the case, presenting a changeover in the observed character of the He I $10830 \mathrm{~A}$ spectral line between between strong-steady absorption and much weaker, variable absorption at the same spectral type location along the giant sequence.

Early observations with the $I U E$ satellite suggested to Linsky and Haisch (1979) that there was a clear separation between stars showing or not showing ultraviolet emission lines corresponding to Transition Region (TR) gas (at $10^{5} \mathrm{~K}$ ) in their outer atmospheres. More intensive observations (Simon et al. 1983; Haisch 1987) appear to support the initial impression. This dividing line, labelled $\mathrm{T}$ in Fig. 1, occurs near the coronal dividing line. This coincidence makes physical sense if the outer atmospheric structure of the Sun, where the TR underliés coronal gas, is used as the archetype. There is an important group of stars, the K-type bright giants ("hybrids"), which violate this division by showing transition region emission lines and strong mass loss (see below), but these are now associated with older, helium core-burning objects, evolving blueward (Brown 1986).

What is remarkable about these two essentially coincident dividing lines is that they occur at the base of the nearly vertical ascent in luminosity, up the red giant branch, for 
1-2 solar mass stellar models, at the point of predicted first dredge up (Iben 1981) and an observed rapid change in $\mathrm{C} / \mathrm{N}$ surface abundances (Brown 1987). The additional lines in Figure 1 can be viewed in terms of additional envelope and atmospheric responses to interior changes.

The three remaining dividing lines illustrated in Figure 1 can be viewed in terms of the effects of all the previously outlined physics. Two of the lines (marked $\mathrm{C}$ and $\mathrm{M}$, respectively) relate to the observed asymmetry in the emission cores of the $\mathrm{Ca}$ II and $\mathrm{Mg}$ II resonance lines. These chromospheric features show a "doubly reversed" emission core as a consequence of their formation under optically thick conditions (cf. Mihalas 1970). Static atmospheres produce symmetric doubly reversed profiles. Although there are ambiguities in interpreting the profile asymmetry, it is reasonable to ascribe asymmteries to systematic velocity fields in the chromosphere. Stencel (1978), and Stencel and Mullan (1980) found, statistically, that the emission core asymmetries changed systematically from the solar-like blue peak dominated shape to a red peak dominated shape, for stars cooler than along the coronal dividing line. The offset between these dividing lines may reflect their relative optical depths ( $\mathrm{Mg}$ II being greater). The Mg II survey was done early in IUE's lifetime and should be re-examined with more complete data. Earlier, Reimers (1977) had identified an additional locus above which stars exhibited blueshifted lines due to cool, neutral gas ascribed by Deutsch (1956) to circumstellar shells (labelled CS in Fig. 1). Taken together, these dividing lines suggest a transition from the circulation patterns observed in the solar chromosphere, to predominantly outflow velocities involving increasing amounts of cool material. Indeed, mass loss derivations suggest a gradual increase in the same direction, consistent with the simple velocity field change concept outlined here.

The third and final division is for stars which show evidence for dust production in their infrared spectra. Woolf and Ney (1969) pointed out the match between the 9.7 micron spectral emission feature in a number of cool giants and the infrared spectrum of minerals like olivine (e.g. $[\mathrm{Mg}, \mathrm{Fe}]_{2} \mathrm{SiO}_{4}$ ). The working hypothesis is that such stars produce silicate dust grains in association with enhanced mass loss. Numerous observations support this view (cf. Volk and Kwok 1987). Merrill and Stein (1986), citing unpublished work by Gilman and Woolf, described this dust formation locus (labelled D in Figure 1). IRAS data largely agrees with its placement. The dust dividing line largely coincides with the onset of pulsation along the red giant branch: stars cooler than M4-M5 show marked, periodic light variation (cf. Brugel, this volume). Many of these are, of course, Mira variables. Jura (1986) has demonstrated a strong correlation between pulsation, dust production and high rates of mass loss among such stars.

Also largely coincident with stars above this dividing line is the occurrence of stellar masers (Olnon et al. 1980; Bowers 1985). Masers are observed to occupy a large volume, and indicate the circumstellar chemistry associated with dust production and heavy mass loss. According to Allen (this volume), only $\mathrm{R} \mathrm{Aqr}$ and H1-36 among Symbiotics show any maser emission ( $\mathrm{SiO}$ ). Apparently, tidal interaction can disrupt $\mathrm{H}_{2} \mathrm{O}$ and $\mathrm{OH}$ maser occurance among Symbiotic binary stars.

The environments of cool stars are strongly affected by their ongoing mass loss processes. Recently, it has been recognized in the IRAS data that several late-type objects possess spatially extended far infrared emission (Hacking et al. 1985). R CrB shows an 18 
arc minute shell (Gillett et al. 1986). Stencel et al. (1987) have discussed the evidence for similar shells surrounding a number of visually bright red supergiants. Such cool shells (at roughly $50^{\circ} \mathrm{K}$ ) are simply interpreted in terms of the remnants of long term mass outflow, implying several tenths of a solar mass lost over timescales of $10^{5}$ years.

The application of Charge Coupled Devices (CCDs) to imaging planetary nebulae has produced an astonishing array of structures associated with former red giant stars. Balick (1987) has summarized some of the discoveries, including a surprisingly high frequency of bipolar symmetries and multiple shells. The global symmetry argues for more importance of rotation and/or magnetic fields than usually assumed at these advanced stages of evolution (Gray 1986), particularly when helium shell flashes are occuring.

Finally, overlaid on all of this, is the fact of variability on almost every observed timescale, even among the non-pulsating stars. The extent of data varies, but it is clear that the mass loss process is episodic in some stars. Chromospheric emission profiles and associated circumstellar features vary on a few month timescale (Brosius et al. 1985). Dust emission is observed to vary in intensity from one season to the next (Bloemhoef et al. 1985). SiO maser cores closest to the star come and go on a few hundred day timescale (McIntosh 1987). Interpolating between high resolution observations of the turbulent solar surface and the bipolar maser maps (Chapman and Cohen 1987) ought to caution anyone who imagines that static, homogeneous models of stellar surfaces are adequate approximations.

Although I have not dealt much with abundance and isotopic effects, these too are important clues to evolution-driven atmospheric changes. Nor have I mentioned polarimetry, which will prove increasingly important as the technique matures (cf. Doherty 1986; Magalhaes, this volume). The challenge before us is to place all of the above information concerning the environments of single cool stars into the context of interacting binaries, like the Symbiotics.

\section{Model Outer Atmospheres}

In order to interpret the dividing lines, we need a realistic magnetohydrodynamic description of the outer atmospheres of cool stars. There are several good references to developments in model outer atmospheres, including the recent series of Cool Star Workshops published by Springer-Verlag, and the IUE Conferences published by NASA and ESA. These can serve as initial guides to the literature. Before any realistic model atmosphere can be constructed, a knowledge of fundamental parameters is required in order to set correct boundary conditions.

In terms of model stellar atmospheres, the most well determined case is probably the Sun (Vernazza et al. 1981). For evolved stars, the most prolific sources of new models have been the Indiana (Johnson) and Texas-Stockholm groups (Lambert-Gustafsson). There are still disagreements of several hundred degrees Kelvin in the temperature scale for M giants (cf. Johnson 1987) and the determination of stellar gravities for red giants desperately needs work. Models for chromospheres and transition regions have been developed by Ayres with semi-empirical multi-level radiative transfer calculations, and by Jordan using emission measure techniques. All of these models emphasize the commonality of physics 
among solar-like stars. In particular, for the lower gravity stars, an increased mass column density above the base of the chromosphere is implied, consistent with the observation that stellar winds involve more matter flux in such stars. Despite the dividing lines, there is not enough known about the winds of coronal giants to indicate whether there is a smooth or rapid increase in mass loss rates between the thin, hot, fast coronal winds and the thick,

slow non-coronal ones. In addition to the observations, some of the improvements being made to atmospheric models involve inclusion of sphericity, comoving frame methods and more complete treatments of atomic and molecular opacities. In addition, new diagnostics continue to be developed for temperature and density (cf. Feldman 1981; Lennon, et al. 1985).

An excellent review of the possible mass loss mechanisms for cool stars has been given by Holzer and MacGregor (1985). There are four major processes: thermal pressure gradient; Alfven waves; pulsation, and, radiation-driven dust. The thermal pressure gradient is the Parker mechanism, operative in solar-like coronae. Alfven waves are important in the solar wind and may be crucial to understanding most stellar winds. "Molecular catastrophes", as proposed by Muchmore et al. (1987) can help alter the outer atmospheric opacity for stars above the Dust onset line, and give rise to masers and dust formation. Careful evaluation of the optical depths and geometry in extended atmospheres indicates that the radiation force in extended atmospheres does not reach its maximum for several stellar radii above the surface (Abbott and Friend 1986). This suggests that chromospheric gas may persist below substantial dust shells. Among Miras, pulsation can levitate matter to the necessary altitude for dust formation to begin. Because there is measureable mass loss for non-coronal stars below the Dust onset line in Fig. 1, and for stars warmer than Mira variables, then pulsation, dust and probably thermal pressure gradients can be ruled out as causes of mass loss among such stars. Alfven waves hold great promise, but magnetic fields are required. Recently, Cohen (1987) has observed Zeeman splitting in the $\mathrm{OH}$ maser lines of the red supergiant VX Sgr, suggesting surface magnetic fields of several Gauss.

\section{Behavior of Red Giants/Supergiants in Binary Systems}

Space does not permit a complete elaboration of the implications of placing a cool star and its naturally occuring circumstellar environment within the tidal influence of a hot companion star. This is in fact part of the purpose of this meeting.

To provoke some discussion and thought, I have placed in Table 1 a cursory set of possibilities. Any comparison of a Symbiotic's UV and X-ray output to a single late type giant immediately reveals that the Symbiotic output is greatly enhanced. This is why EXOSAT, HEAO-2 and IUE could observe a number of visually faint Symbiotics compared with rather few visually bright single red giants. The ouput is probably not standard chromospheric or coronal emission. The possible causes for this enhancement include the illuminated nebular material over a large apparant surface area, possibly some shock excited gas as is seen in the $\zeta$ Aur systems (Ahmad et al. 1983), or even because of magnetic enhancement of the output as a result of tidal synchronization analogous to the short period RS CVn stars (Rutten and Schrijver 1987; Savonije and Papaloizou 1985). Densities derived using emission line diagnostics (Nussbaumer and Stencel 1987) tend to 
Table 1: Comparison of Cool Star Environments

\begin{tabular}{|lcr|} 
Observable & Binary-vs-Single & Physical Cause \\
\hline \hline UV,X emission & much enhanced & $\begin{array}{r}\text { not chromospheric } \\
\text { prob. Stromgren, } \\
\text { shock structure? } \\
\text { rotation-activity? }\end{array}$ \\
\hline $\begin{array}{l}\text { mass loss, } \\
\text { dust production }\end{array}$ & $\begin{array}{c}\text { tidally } \\
\text { constrained }\end{array}$ & $f(\mathrm{P})$, plus disk IR \\
\hline variability & same? & internally driven \\
\hline \hline
\end{tabular}

be somewhat larger than determined for single cool giant chromospheres (Lennon et al. 1985).

Second, mass loss is enhanced by dust production in the circumstellar environment. Building on a suggestion by Allen (1983), Kenyon et al: (1986) have argued on the basis of IRAS data that D-type Symbiotics are distinguished from their S-type counterparts by heavy reddening of the Mira variable member, with the hot component lying outside this dust shell. I would like to take this one step further and argue that the distinction between Dusty and Stellar Symbiotics is largely due to orbital separations. The longer period systems provide the volume necessary for the cool component to run through more of its intrinsic evolution prior to tidal interaction. This provides the time for Mira characteristics to develop, including substantial dust production and increase mass loss. That this occurs is suggested by the influence of orbital separation on derived mass loss rate among the $\zeta$ Aur binaries. Dupree and Reimers (1987) summarize such rates for a set of these objects, but the trend in mass loss rate is consistent with $\mathrm{P}^{2 / 3}$ and hence orbital separation. The occurrence of $\mathrm{SiO}$ masers is generally on scales of $10^{15} \mathrm{~cm}$ among single stars, and their unimpeded development in Symbiotics would imply very long periods.

Finally, we have mentioned that mass loss is episodic in some of the single stars. Assuming this characteristic is unmodified in a binary situation, the temporal variation helps understand some of the fine structure in Symbiotic light curves. Short term changes could reflect the stochastic production of dust clouds ejected and intercepting the accretion field of the hot companion, while the slow nova characteristics could reflect the result of helium shell flashes producing planetary nebula shells late in the life of Miras. Perhaps, simply speaking, the dust free s-types evolve to their Roche limits on the first ascent of the red giant branch, the d-prime types while on the Helium burning main sequence and the d-types while on the AGB.

I wish to thank the organizers of this Colloquium for their support, encouragement and hospitality. I also acknowledge NASA grants NAG5-816 and JPL 957632 for partial support in this work. It is a pleasure to thank Joseph Pesce for additional help in the prepa- 
ration of the manuscript. I hope the conference will bring about an harmonic convergence of ideas about Symbiotics.

\section{REFERENCES}

Abbott, D. and Friend, D. 1986 Ap.J. 311, 701.

Ahmad, I., Chapman, R. and Kondo, Y. 1983 A\&A 126, L5.

Allen, D. 1983 M.N.R.A.S. 204, 113.

Ayres, T., et al. 1981 Ap. J., 250, 293.

Balick, B. 1987 Astron. J. in press (October).

Bloemhoef, E., Danchi, W. and Townes, C. 1985 Ap.J. 299, L37.

Bowers, P. 1985 in Mass Loss from Red Giants, ed. M. Morris and B. Zuckerman (Reidel; Dordrecht), p.189.

Brown, A. 1986 Adv. Space Res. 6, 195.

Brown, J. 1987 Ap.J. 317, 701.

Brosius, J., Mullan, D. and Stencel, R. 1985 Ap.J. 288, 310.

Carpenter, K., Brown, A. and Stencel, R. 1985 Ap.J. 289, 676.

Chapman, J. and Cohen, R.J. 1986 M.N.R.A.S. 220, 513.

Cohen, R.J. 1987 M.N.R.A.S. 225, 491.

Deutsch, A. 1956 Ap.J. 123, 210.

Doherty, L. 1986 Ap.J. 307, 261.

Dupree, A. and Reimers, D. 1987 in Exploring the Universe with the IUE Satellite, ed. Y. Kondo et al. (Dordrecht; Reidel), p. 321.

Feldman, U. 1981 Physica Scripta 24, 681.

Gondoin, P., Mangenay, A. and Praderie, F. 1987 A $\& A$ 174, 187.

Gillett, F., Backman, D., Beichman, C. and Neugebauer, G. 1986 Ap.J. 310, 842.

Gray, D. 1986 Adv. Space Res. 6, 161.

Gray, D. and Toner, C. 1986 Ap.J. 310, 277.

Hacking, P., al. 1985 Publ. Astr. Soc. Pacific 97, 616.

Haisch, B. 1987 in The Fifth Cambridge Workshop on Cool Stars, Stellar Systems and the Sun, ed. J. Linsky and R. Stencel, (Springer-Verlag; Heidelberg), in press.

Haisch, B. and Simon, T. 1982 Ap.J. 263, 252.

Iben, I. 1981 in The Physics of Red Giant Stars, ed. I. Iben and A. Renzini (Reidel; Dordrecht), p.25.

Johnson, H. 1987 in The Fifth Cambridge Workshop on Cool Stars, Stellar Systems and the Sun, ed. J. Linsky and R. Stencel, (Springer-Verlag; Heidelberg), in press.

Judge, P. 1986 M.N.R.A.S. 221, 119.

Jura, M. 1986 Irish Astron. Journal 17, 322.

Kenyon, S. 1986 The Symbiotic Stars (University Press; Cambridge).

Kenyon, S., Fernandez-Castro, T. and Stencel, R. 1986 Astron. J. 92, 1118.

Lennon, D., Dufton, P., Hibbert, A. and Kingston, A. 1985 Ap.J. 294, 200.

Linsky, J. and Haisch, B. 1979 Ap.J. 229, L27.

Holzer, T. and MacGregor, K. 1985 in The Physics of Red Giant Stars, ed. I. Iben and A. 
Renzini (Reidel; Dordrecht), p.25.

McIntosh, G. 1987 Dissertation, University of Massachusetts.

Merill, K. and Stein, W. 1976 Publ. Astr. Soc. Pacific 88, 285.

Mihalas, D. 1970 Stellar Atmospheres, Freeman, San Fransisco.

Muchmore, D., Nuth, J. and Stencel, R. 1987 Ap.J. 315, L141.

Nussbaumer, H. and Stencel, R. 1987 in Exploring the Universe with the IUE Satellite, ed.

Y. Kondo et al. (Dordrecht; Reidel), p. 203.

O'Brien, G. and Lambert, D. 1986 Ap.J.Suppl. 62, 899.

Olnon, F., Winnberg, A., Matthews, H. and Schultz, G. 1980 A 8 A Suppl. 42, 119.

Reimers, D. $1977 A B A$ 57, 395.

Rutten, R. and Schrijver, C. 1987 A 8 A 177, 155.

Savonije, G. and Papaloizou, J. 1985 in Interacting Binaries, ed. P. Eggleton and J. Pringle (Dordrecht: Reidel), p. 83.

Simon, T., Linsky, J. and Stencel, R. 1983 Ap.J. 257, 225.

Stencel, R. 1978 Ap.J. 223, L37.

Stencel, R. and Mullan, D. 1980 Ap.J. 238, $221 \& \mathbf{2 4 0}, 718$.

Stencel, R. 1980 in Cool Stars, Stellar Systems and the Sun, ed. A. Dupree; Smithson. Astrophys. Obs. Special Report 389, p. 183.

Stencel, R., Pesce, J. and Bauer, W. 1987 Astron. J. submitted.

Vernazza, J., Avrett, E. and Loeser, R. 1981 Ap.J. Suppl. 45, 635.

Volk, K. and Kwok, S. 1987 Ap.J. 315, 654.

Woolf, N. and Ney, E. 1969 Ap.J. 155, L183.

Postscript: Two additional important articles which may interest the reader are ones by R. Gilliland 1985 Astrophys. Journ. 299, 286 entitled "The Relation of Chromospheric Activity to Convection, Rotation and Evolution off the Main Sequence", and by S. Drake, J. Linsky and M. El itzur 1987 Astron. Journ. (in press) entitled "A Radio Continuum Survey of the Coolest M and C Giants". 